\title{
puente de hormigón pretensado sobre el río Caroní
}

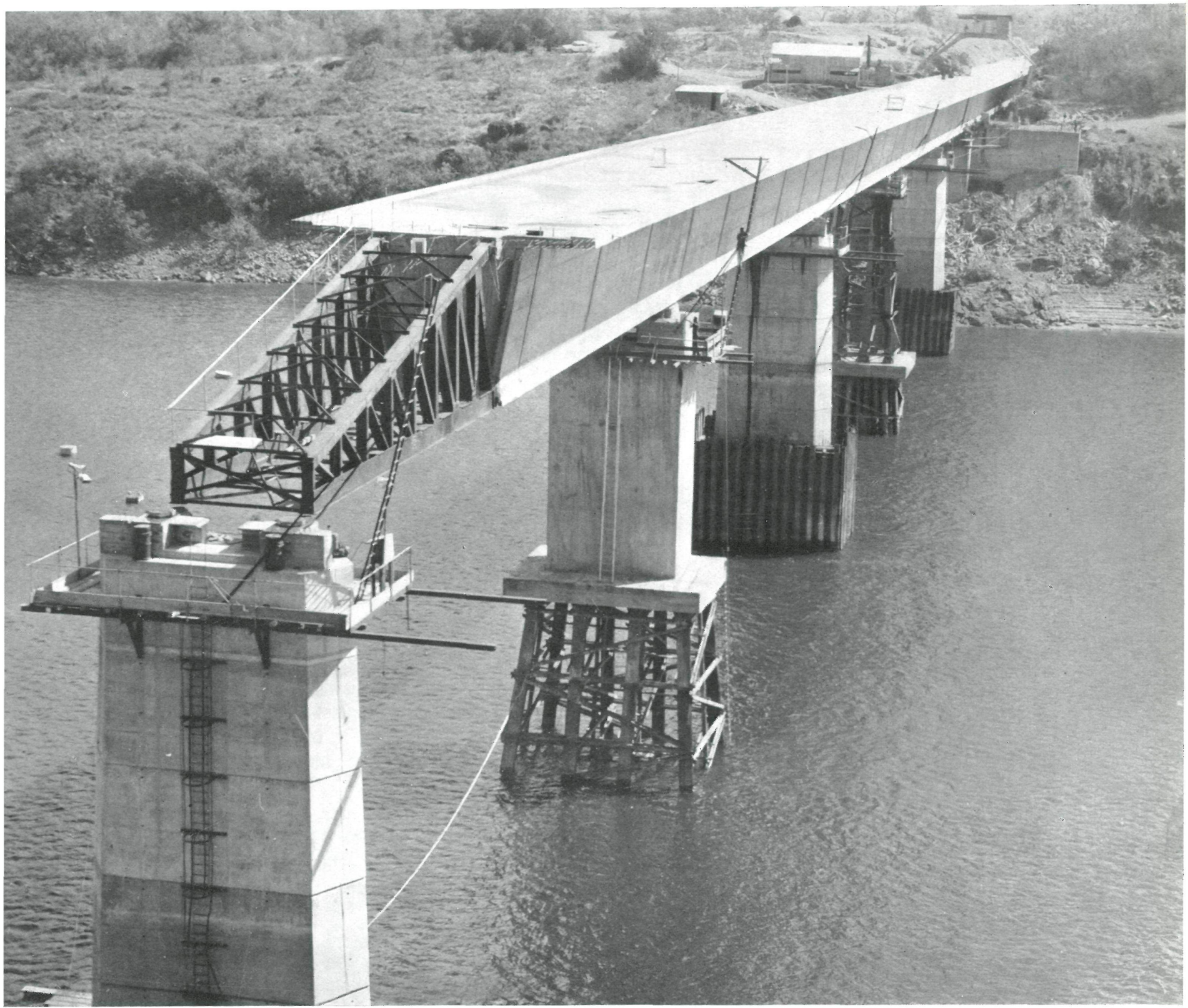

Corrimiento del tablero.

Este puente consta de una serie de tramos porticados de luces máximas $(96 \mathrm{~m})$, con longitud total de unos 500 metros. Las pilas o soportes han presentado cierta dificultad en los tramos correspondientes al río, pues han sido necesarias ataguias formadas por células de tablestacas que han servido de encofrados perdidos para hormigonar los cimientos. E conjunto del tablero está constituido por una viga-cajon, de hormigón pretensado. La particularide hastivido en 50 trozos prefabricados en un taller próximo al puente y alineado con el eje del mismo, que ha servido de rampa para el traslado y corrimiento a su posición definitiva. Para esta delicada y notable operación se ha utilizado el neopreno, que presenta un coeficiente de rozamiento muy pequeño al deslizar sobre acero. Para empujar al pesado tablero hacia su posición definitiva se han empleado gatos hidráulicos. Terminadas todas estas operaciones, se procedió a rectificar el trazado de los paquetes de cables del pretensado que provisionalmente se habían tesado antes de proceder al corrimiento. Estos paquetes de cables van adosados, cada uno, a la parte interior de los tabiques laterales de la viga-cajón que constituye la estructura del tablero. 
sección longitudinal

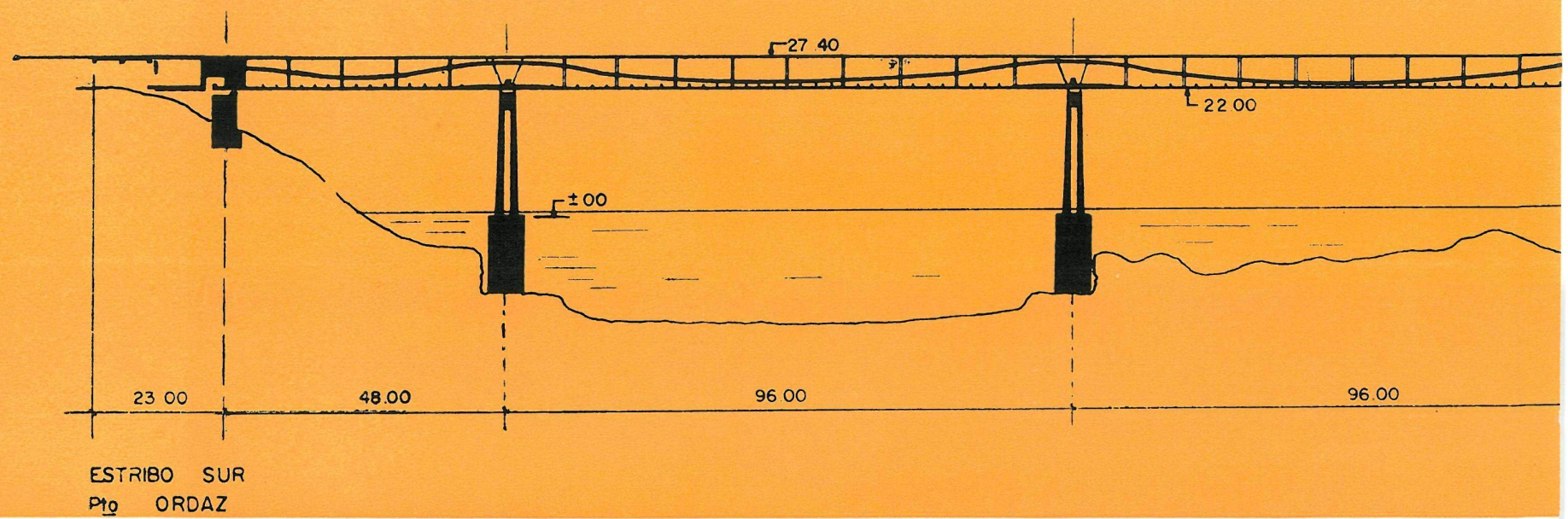

\section{Prelíminumes}

La "Corporación Venezolana de Guayana", organismo autónomo del Estado, ha patrocinado la construcción del puente sobre el río Caroní, a $4 \mathrm{~km}$ de su confluencia con el río Orinoco. El objeto en esta obra es el de unir las poblaciones de San Félix y Puerto Ordaz, centro minero y siderúrgico del país.

El proyecto fue adjudicado al ingeniero consultor Prof. Dr. Leonhardt, de Stuttgart, y la construcción, al Consorcio formado por las empresas Tuven C. A., de Venezuela, y C. Baresel A. G. y E. D. Zublin A. C., de Alemania.

El procedimiento constructivo es único en el mundo hasta la fecha y abre, sin lugar a duda, un nuevo camino en la prefabricación y construcción de grandes puentes. El método tiene el doble mérito de ser: muy ingenioso y de gran sencillez. Este puente, de hormigón pretensado, es continuo a lo largo de $480 \mathrm{~m}$ y su peso total es de 10.000 toneladas.

\section{Descripcidon del proyecto}

El río Caroní recoge las aguas de una hoya del interior de las selvas de la Guayana venezolana y, por consiguiente, su régimen es el característico de todos los grandes ríos de las zonas tropicales. En la época de intensas lluvias su caudal llega a los $16.000 \mathrm{~m}^{3} / \mathrm{s}$. La profundidad media del río es de $21 \mathrm{~m}$ en el emplazamiento del puente. La velocidad máxima del agua es de $5 \mathrm{~m} / \mathrm{s}$. Estos datos dan una idea de las dificultades encontradas en la ejecución del puente.

El fondo del cauce está constituido por una roca granítica de excelentes cualidades, meteorizada únicamente en la capa superficial.

Cerca de la margen izquierda se encuentra un surco, de sección triangular, paralelo al eje del río, relleno de arenas de aluvión, y sobre el cual no era prudente apoyar las pilas del puente. Esta circunstancia obligó a fijar las luces máximas del mismo en $96 \mathrm{~m}$ en los cuatro tramos centrales y $48 \mathrm{~m}$ en los dos de acceso.

El régimen del río, así como las características del fondo, condujeron al estudio de una solución original basada en la prefabricación y montaje sin entramados auxiliares.

La superestructura del puente la constituye una viga continua, cajón, de hormigón pretensado, de sección constante, con un ancho útil de calzada de $8,50 \mathrm{~m}$ y dos bordillos de $0,60 \mathrm{~m}$. El canto de la viga es de $5,40 \mathrm{~m}$ y su longitud salva seis tramos.

A cada lado del tablero, y en su parte inferior, salen en voladizo los dos andenes, de 3,0 $\mathrm{m}$ de anchura, prefabricados, que posteriormente se solidarizan con la estructura del tablero por medio de cables transversales del pretensado.

Debido a este emplazamiento, los andenes estarán protegidos por la sombra de la estructura, y por ser independientes de la calzada se evitan posibles accidentes.

La losa superior del tablero es de hormigón armado, pretensado transversalmente con cables que trabajan con un esfuerzo de $33 \mathrm{t}$ cada uno y espaciados a 1,50 metros.

Las pilas son huecas, de hormigón armado y de sección variable. 


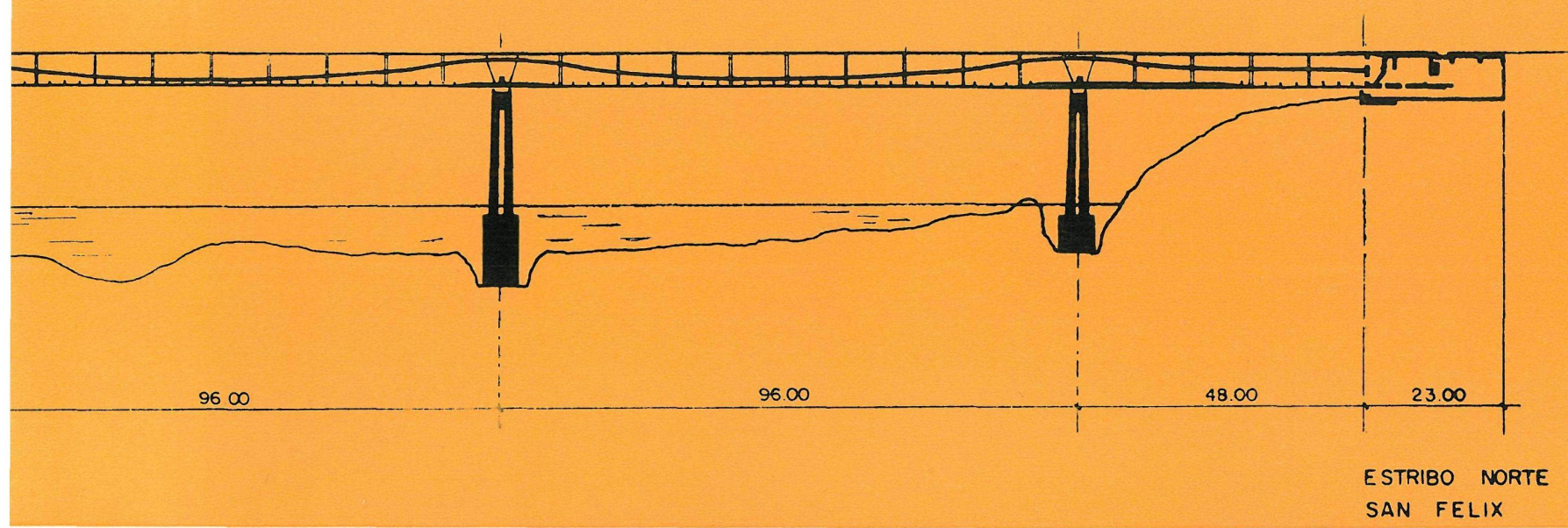

El puente constituye una estructura continua, de $500 \mathrm{~m}$ de longitud, y se apoya sobre una articulación fija en el estribo de Puerto Ordaz y sobre rodillos "Corroweld» en las pilas y estribo de San Félix. El rodillo más alejado de la articulación fija permite una dilatación longitudinal del puente de hasta 13 centímetros.

\section{Procedimiento constructivo}

Los cimientos de las pilas se ejecutaron aprovechando el período de sequía, y a una profundidad variable de 9 a $13 \mathrm{~m}$. Primeramente se formó una ataguía de tablestacas metálicas, guiadas con una plantilla solidarizada a dos gabarras convenientemente ancladas a las orillas y a varias boyas.

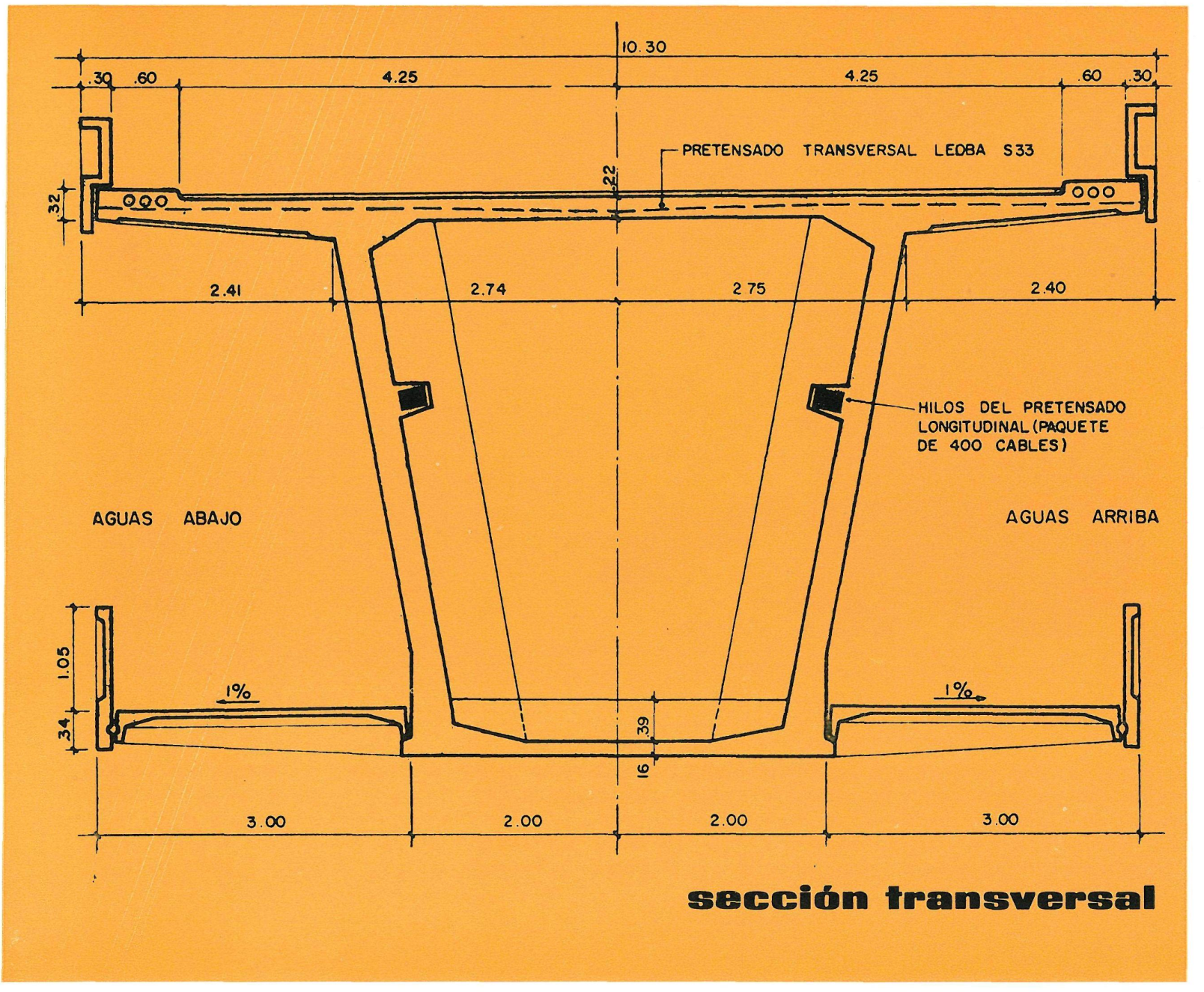




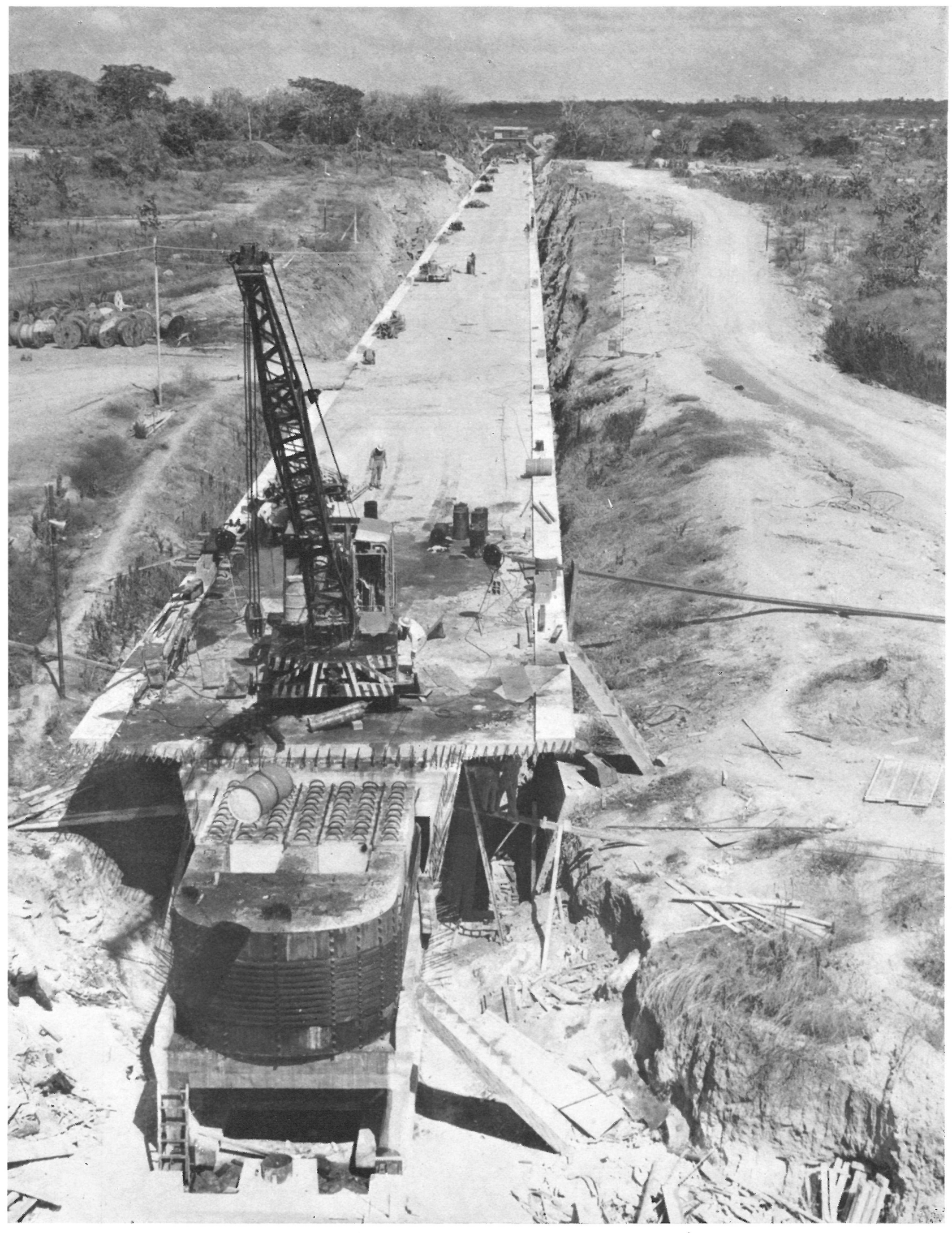

El tablero an tes de ser co-

Se limpió la roca meteorizada superficialmente con voladuras debajo del agua, y a continuación se llenó la ataguía con un hormigón, tipo "Prepakt», hasta una altura variable de 3 y $7 \mathrm{~m}$, según las pilas. Encima del bloque de hormigón "Prepakt)» se construyó una placa o solera fuertemente armada, de 2,50 $\mathrm{m}$ de espesor, destinada a repartir uniformemente los esfuerzos transmitidos por el fuste. Esta losa se ejecutó previo agotamiento del área de trabajo, ya que la presión hidrostática, de $5 \mathrm{~m}$, la podía resistir el tables- 
tacado. El cimiento así constituido tiene una sección rectangular de $6 \times 10 \mathrm{~m}$. El tablestacado se utilizó como encofrado perdido.

La superestructura, de sección en forma de cajón, se prefabricó en 50 elementos independientes, de 9,20 metros de longitud, pudiendo emplearse el mismo encofrado metálico para todos ellos.

Todos los elementos son iguales, salvo la tabla inferior, que es más gruesa en los elementos que absor. berán momentos negativos. En primer lugar se hormigonó la tabla inierior y, una vez endurecida, se colo. caron los encofrados laterales y el central, el cuaí se apoyaba en la tabla inferior, continuando después el resto del elemento.

De esta forma, todas las instalaciones para el hormigonado eran fijas, con lo que se consigue un excelente rendimiento y control del hormigón.

Terminado un elemento, se le hacía deslizar sobre dos pistas de madera dura y engrasada tirando de dos cabrestantes anclados al estribo de Fuerto Ordaz. De esta forma se fueron colocando estos elementos, uno tras otro, sobre el terreno y en la prolongación del eje del puente. Los 50 elementos quedaron apoyados sobre dos pistas de madera, horizontales, situadas debajo de las almas del cajón. Posteriormente, se reilenaron las juntas, de $40 \mathrm{~cm}$, formadas entre los elementos, siguiendo un orden estudiado para evitar fisuraciones de origen térmico.

Los cables longitudinales para el pretensado se colocaron, en el interior del cajón, agrupados en dos paquetes y exteriores a las almas.

Dicha colocación anterior se efectuó con ayuda de un carretón porta-bobinas, automotor, proyectado para el efecto. Cada cable, convenientemente guiado, se colocaba sobre una pista de madera respetando el espaciamiento previsto. Se tendieron 400 cables, cada uno de ellos compuesto de siete alambres de $3 \mathrm{~mm}$ de diámetro, de acero, de una resistencia a la rotura de $180 \mathrm{~kg} / \mathrm{mm}^{2}$, dando la vuelta en los dos extremos de la viga continua y anclánđose en un bloque móvil que permitirá el tesado de los mismos. La armadura total de cada sección transversal es, por consiguiente, de 800 cables y proporciona un esfuerzo de pretensado inicial de 4.900 toneladas.

El trazado de cables en esta primera etapa es rectilíneo-en una longitud de $480 \mathrm{~m}$-, para reducir al mínimo las pérdidas por rozamiento. El paquete de cables se colocó ligeramente por encima del centro de gravedad de la sección, con objeto de resistir mejor a las tensiones producidas por el voladizo durante la colocación de la viga cajón.

Cada paquete está formado por 20 capas horizontales, de 20 cables cada una, espaciados a $1 \mathrm{~mm}$ por medio de separadores especiales.

Este espaciamiento es suficiente para permitir un inyectado y recubrimiento final.

Los cables se tesan con ayuda de un batería de 20 gatos hidráulicos, colocados entre el bloque móvil de anclaje y la superestructura. El alargamiento total fue de 3 metros.

El problema principal consistía en colocar en posición definitiva la estructura en forma de cajón, continua, de $480 \mathrm{~m}$ de longitud y de un peso de $10.000 \mathrm{t}$, apoyándose sucesivamente en las pilas. Este método constructivo presenta un interés singular. La dificultad surgía al evaluar las fuerzas de rozamiento que podrían aparecer al emplear dispositivos clásicos, así como la determinación de las fuerzas horizontales que comprometían la estabilidad de las pilas de gran altura.

Esto fue resuelto brillantemente empleando un nuevo tipo de plástico llamado "teflon». El teflon es un hidrocarburo al cual se le han reemplazado algunos átomos de hidrógeno por otros de flúor, y tiene la propiedad de poseer un coeficiente de rozamiento contra el acero extraordinariamente bajo y sin necesidad de lubricante. En Alemania se realizó una serie de ensayos para estudiar el comportamiento del tefión en condiciones análogas a las que tendría al ser empleado como patín deslizante en el corrimiento del puente. Se observó que el coeficiente de rozamiento decrece rápidamente después de la primera utilización y llega hasta un valor de $u=0,002$ para la carga normal de trabajo. También se demostró que este valor es tanto más pequeño cuanto mayor es la presión normal al movimiento, propiedad ésta favorable, dada la magnitud del peso de la superestructura.

Se han previsto cuatro gatos tractores, anclados al estribo de Puerto Ordaz y con una capacidad de tracción total de $600 \mathrm{t}$, suficiente para paliar cualquier aumento accidental del rozamiento. Durante el corrimiento de la estructura se observó que para iniciar el arranque se necesitaba una fuerza de $250 \mathrm{t}$, que descendía a 200 t durante el movimiento.

Las luces de $96 \mathrm{~m}$ en los tramos sobre el río se redujeron a $48 \mathrm{~m}$ por medio de cuatro pilas auxiliares. En la pista de corrimiento del lado de Puerto Ordaz se prepararon estaciones de elevación y deslizamiento cada $48 \mathrm{~m}$. Cada estación, así como cada cabeza de pila auxiliar y definitiva, poseía dos chapas de acero 
cromado, de $50 \mathrm{~mm}$ de espesor, una a cada lado de la superestructura y por debajo de las dos almas del cajón. Estas chapas, de $1,20 \mathrm{~m}$ de longitud, se nivelaron con gran precisión y se anclaron al terreno o a las pilas. Entre cada chapa y la viga del puente se intercaló un patín, compuesto de la siguiente forma: En su parte inferior, un disco de teflon, de $5 \mathrm{~mm}$ de grueso y $60 \mathrm{~cm}$ de diámetro, retenido en una envoltura metálica. Por encima, una capa de caucho neopreno, para permitir al patín acoplarse a las deformaciones y, el todo, envuelto por una caja metálica. Entre el patín y la viga del puente se colocó una lámina de caucho para asegurar la adherencia entre ambos. Ei patín desliza sobre la chapa cromada por el intermedio del disco de tefion.

Los gatos de tracción, situados por parejas a cada lado de la superestructura, tiran de dos barras metálicas que se fijan a las paredes de la viga cajón por medio de grapas a presión.

Al lado de cada estación de corrimiento, es decir, cada $48 \mathrm{~m}$, se colocaron dos pares de gatos hidráulicos debajo de las almas de la viga y destinados a levantar toda la superestructura. El total de gatos ele. vadores fue, por consiguiente, de 44 .

El corrimiento se realizó en las etapas siguientes:

a) Se levantó toda la viga, continua, con los 44 gatos.

b) Se colocaron los patines de teflon en su posición.

c) Se bajó la viga hasta apoyarla sobre los patines.

d) Se accionaron los gatos de tracción avanzando 96 centímetros.

e) Se volvió a levantar la viga, continua, aflojando los patines.

f) Se corrieron los patines hacia atrás a su posición inicial.

g) Se repitieron las operaciones anteriores a partir de c).

Se consiguió una velocidad de avance de $19,20 \mathrm{~m}$ por jornada de diez horas, reservando para la noche el cambio de posición de las grapas de apriete de las barras de tiro.

Todas estas operaciones necesitaron un control riguroso de los niveles de las chapas de apoyo, de las deformaciones de las pilas y flecha de la «cola» de la superestructura. El proyecto sólo toleraba diferencias de nivel de $2 \mathrm{~cm}$ entre puntos distantes $48 \mathrm{~m}$, teniendo en cuenta la gran rigidez de la viga. También se instaló un anemómetro para controlar la velocidad del viento, ya que si ésta pasaba de $15 \mathrm{~m} / \mathrm{s}$, era necesario suspender el corrimiento para evitar desviaciones laterales.

Cada $96 \mathrm{~m}$ se colocaron unas guías laterales formadas por ruedas de caucho, de eje vertical, que podían desplazarse horizontalmente por medio de gatos de mano, con lo cual se podían corregir las desviaciones durante el corrimiento.

Todas las operaciones se dirigieron desde un puesto de mando convenientemente situado y desde el cual se comunicaba, telefónicamente, con todas las estaciones de elevación y deslizamiento, así como con el operario que accionaba los gatos de tracción. Un cuadro con luces de colores indicaba cuándo todas las estaciones estaban con sus patines en posición «atrás» y si estaban actuando los gatos elevadores.

Para reducir el momento flector en el voladizo, la viga va provista de un entramado auxiliar metálico en su extremidad.

El día 2 de enero de 1964 se comenzó el corrimiento del tablero de $10.000 \mathrm{t}$ de peso, para terminar con pleno éxito al apoyarse en el estribo de San Félix el día 8 de febrero.

Una vez el puente en su posición definitiva se procedió a cambiar el trazado de los cables para adoptar la forma óptima en las condiciones de servicio, para lo que se han previsto luces centrales de $96 \mathrm{~m}$. Por ello, fue necesario modificar el trazado inicial, recto, en una serie de poligonales con los puntos altos sobre los apoyos y los bajos en los centros de cada tramo y con los vértices entre apoyos situados en una parábola. Essta operación se realizó, tramo por tramo, empezando desde el extremo opuesto al bloque auxiliar de tesado.

Los cables se desplazan verticalmente, ya hacia arriba o hacia abajo, por medio de gatos hidráulicos que se apoyan en la tabla superior o inferior de la viga y que tiran de cuatro barras, de alta resistencia, unidas a una pieza metálica, rígida, que abraza el paquete de 400 cables. Una vez terminado el desplazamiento en un punto, correspondiente a un vértice de la poligonal, se termina el hormigonado de un marco rigidizador de la viga cajón, que transmite también a la estructura las fuerzas provocadas por el desvío de los cables.

El paso del trazado rectilíneo al poligonal crearía una sobretensión inadmisible en los cables, por lo cual periódicamente, y según un programa cuidadosamente estudiado, se afloja el tesado en el extremo del bloque móvil. 
El nuevo pretensado, así aplicado a la viga continua, hace que ésta se separe de las pilas auxiliares, lo que permite que estas pilas se puedan desmontar a continuación.

Por último, los paquetes de cables se protegen con una inyección de cemento y se solidarizan a las almas de la viga con las armaduras dejadas al exterior a este efecto. Además de la protección contra la oxidación, con ello se consigue aumentar el coeficiente de seguridad a la rotura.

Los cálculos se basaron en las normas de la AASHO para un tren de camiones H20-S16-44.

Con la ayuda de calculadoras electrónicas fue posible hallar las tensiones en un gran número de secciones y en una sucesión móvil durante el corrimiento. La tensión de compresión máxima del hormigón fue de $140 \mathrm{~kg} / \mathrm{cm}^{2}$ en la fase provisional del corrimiento, siendo de $120 \mathrm{~kg} / \mathrm{cm}^{2}$ en las condiciones de servicio.

En la obra se obtuvo una resistencia media de $397 \mathrm{~kg} / \mathrm{cm}^{2}$ a 10 veintiocho días y con probetas cilíndricas.

Entramado auxiliar para apoyo durante el corrimiento.

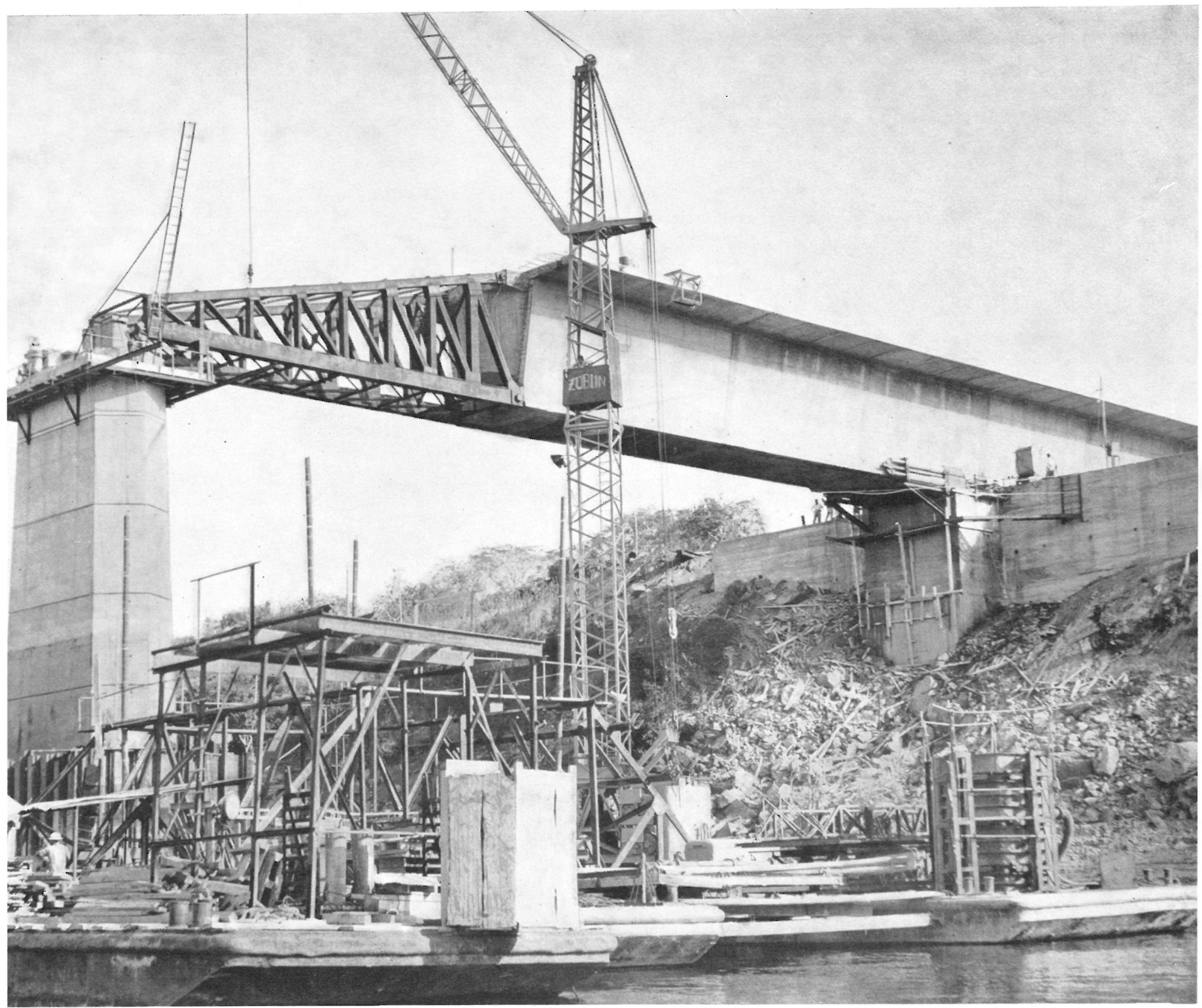




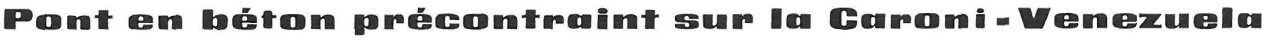

Alvaro Fernández, ingénieur inspectour.

Ce pont, composé d'une série de travées en portique de portées maximales $(96 \mathrm{~m})$, est d'une longueur totale d'environ $500 \mathrm{~m}$.

Les piles ont présenté quelque difficulté dans les travées correspondantes à la rivière. Des batardeaux, formés par des cellules de palplanches, ont servi de coffrages perdus pour le bétonnage des fondations.

L'ensemble du tablier est formé par une poutre-caisson, en béton précontraint.

Les particularités les plus remarquables de cet ouvrage sont les méthodes constructives adoptées pour sa réalisation. La longueur totalo du tablier a été divisée en 50 éléments préfabriqués dans un atelier à proximité et dans l'axe de l'ouvrage et qui a servi de rampe pour leur transport et mise en place définitive.

Pour cette délicate et remarquable operation on a utilisé le néoprène qui présente un très faible coefficient de frottement Des vérins hydrauliques ont été utilisés pour pousser le lourd tablier et le mettre en place.

Une fois toutes ces opérations terminées, on a rectifié l'alignement original des câbles de la précontrainte qui avaient été provisoirement et partiellement mis en tension avant de procéder à la mise en place. Ces câbles sont tous fixés contre les parois latérales de la poutre-caisson qui forme la structure du tablier.

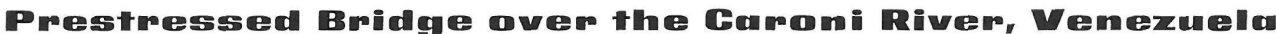

Alvaro Fernández, inspector engineer

This bridge consists of a series of portal frames, of $96 \mathrm{~m}$ span each, making up a total length of about $500 \mathrm{~m}$

The piles over the river have caused certain difficulties, and ît became necessary to build a number cofferdams with sheetpiling, which became permanent formwork for the concrete foundations.

The deck is a box section beam, of prestressed concrete.

The most notable aspect of this structure is the constructive method. The total length of the deck was divided into 50 sections, each of them prefabricated at a nearby workshop. Each section was aligned along the deck axis: the deck already which has a very low friction coefficient, on sliding over steel.

To push the heavy table sections to their final position use has been made of hydraulic jacks.

After these initial operations were completed, the groups of prestressed cables were correctly aligned: these cables had been provisionally stretched, during the process of putting the deck sections together. These groups of cables are attached to the inner surface of the side walls of the box sections of the deck.

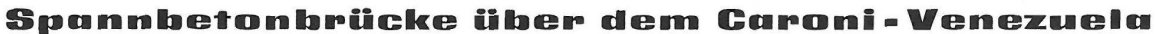

Alvaro Fernández, Ingenieur.

Diese Brücke besteht aus einer Reihe von portalähnlichen Abschnitten mit grössten Spannweiten (96 m) und einer Gesamtlänge von ungefähr $500 \mathrm{~m}$.

Der Bau der Brückenpfeiler für den Abschnitt über dem Fluss brachte einige Schwierigkeiten mit sich, da für die Betonierung der Fundamente die Anwendung von Holzspundwänden als verlorene Schalung notwendig war.

Die gesamte Brückenplatte besteht aus einem Kastenträger aus Spannbeton.

Das Bemerkenswerte an dieser Brücke ist die Art, wie man sie gebaut hat. Die Gesamtlänge der Brückenplatte wurde in 50 vorfabrizierte Teile eingeteilt, die man direkt neben der Brücke in einer Werkstatt herstellte, die unmittelbar eine Fortsetzung der Brückenachse bildetc und gleichzeitig als Rampe zum Transport der einzelnen Teile an die Einbaustelle diente.
Für die Durchführung dieser schwierigen Operation hat man Neopren verwendet, das einen sehr niedrigen Reibungskoeffizienten auf Stahl besitzt.

Um die Brückenplatte in ihre endgültige Lage zu bringen, hat man hydraulische Aufzüge verwendet.

Nach Beendigung der oben beschriebenen Operation, wurden die Spannkabel in ihre richtige Lage gebracht, da man sie vor dem Verschieben der einzelnen Teile nur provisorisch vorgespannt hatte. Jedes einzelne Spannkabel verläuft im Innern der Seitenwände des Kastenträgers, aus dem die Brückenplatte besteht. 